\title{
Author Correction: Measuring the impact of COVID-19 vaccine misinformation on vaccination intent in the UK and USA
}

Sahil Loomba (1), Alexandre de Figueiredo (1), Simon J. Piatek, Kristen de Graaf and Heidi J. Larson (1)

Correction to: Nature Human Behaviour https:/doi.org/10.1038/s41562-021-01056-1, published online 5 February 2021.

In the version of this article initially published, the subscript for the gamma element in equation (7) should have been $k$ rather than $j$; i.e., it should have read $\gamma_{k}^{g}$. The error has been corrected in the PDF and HTML versions of this article.

Original:

$$
\begin{aligned}
Y(i) \mid(G(i) & =g, W(i), X(i)) \\
& \sim \text { OrderedLogistic }\left(\beta_{W}^{g} \sum_{j=1}^{W(i)-1} \delta_{j}^{g}+\sum_{j=1}^{5} \sum_{k=1}^{5} \beta_{j}^{g} X_{j k}(i) \gamma_{j}^{g},\left(\alpha_{1}^{g}, \alpha_{2}^{g}, \alpha_{3}^{g}\right)\right)
\end{aligned}
$$

Corrected:

$$
\begin{aligned}
Y(i) \mid(G(i) & =g, W(i), X(i)) \\
& \sim \text { OrderedLogistic }\left(\beta_{W}^{g} \sum_{j=1}^{W(i)-1} \delta_{j}^{g}+\sum_{j=1}^{5} \sum_{k=1}^{5} \beta_{j}^{g} X_{j k}(i) \gamma_{k}^{g},\left(\alpha_{1}^{g}, \alpha_{2}^{g}, \alpha_{3}^{g}\right)\right)
\end{aligned}
$$

Published online: 8 July 2021

https://doi.org/10.1038/s41562-021-01172-y

๑ The Author(s), under exclusive licence to Springer Nature Limited 2021 